\title{
Efeito analgésico de longa duração da dipirona sobre a hiperalgesia persistente induzida pela constrição do nervo ciático em ratos: participação do óxido nítrico
}

\author{
Fábio José Reis*, Noeli Pereira Rocha
}

Departamento de Farmacologia, Instituto de Biociências, IBB/UNESP, Botucatu - SP

\section{*Correspondência:}

F. J. Reis

Departamento de Cirurgia e Ortopedia

Faculdade de Medicina - UNESP

18618-970 Botucatu - SP, Brazil

E-mail: reisfj@yahoo.com.br
O efeito analgésico de longa duração da dipirona foi avaliado em um modelo de dor neuropática assim como a participação da via óxido nítrico-GMPc neste mecanismo analgésico. Uma única administração intraplantar de dipirona $(80 \mu \mathrm{g})$, no $14^{\circ}$ dia após a instalação da hiperalgesia neuropática induzida pela constrição do nervo ciático exerceu um efeito analgésico, significativo e de longa duração. A inibição da óxido nítrico sintetase com L-NAME (50 ou $100 \mu \mathrm{g} / \mathrm{pata}$ ), ou do óxido nítrico (NO) endógeno com hemoglobina (10 ou $30 \mu \mathrm{g} /$ pata), bloquearam o desenvolvimento do efeito analgésico da dipirona. A L-arginina (500 $\mu \mathrm{g} / \mathrm{pata})$ reverteu o efeito do L-NAME. Cloreto de metiltionínio (azul de metileno) (500 $\mu \mathrm{g} /$ pata), ODQ (50 $\mu \mathrm{g} /$ pata) (bloqueadores da guanilil ciclase) ou glibenclamida (100, 200 ou $300 \mu \mathrm{g} /$ pata) (bloqueador de canais de $K^{+}$sensiveis ao ATP) inibiram o efeito analgésico da dipirona. O nitroprussiato de sódio administrado no $14^{\circ}$ dia após a instalação da hiperalgesia neuropática também exerceu efeito analgésico de longa duração, semelhante ao observado com a dipirona. Sugerimos que a ação analgésica periférica e de longa duração da dipirona, neste modelo experimental, ocorra devido a provável dessensibilização dos nociceptores, envolvendo a via óxido nitrico - GMPc e canais de $K^{+}$sensiveis ao ATP.
Unitermos

- Hiperalgesia neuropática

- Constrição do nervo ciático

- Dipirona

- Óxido nítrico

\section{INTRODUÇÃO}

A dor neuropática ou crônica pode ser resultante de doenças ou injúrias que acometem diretamente o sistema nervoso periférico, assim como a medula espinhal ou o sistema nervoso central (Devor, Seltzer, 1999). O dano ao tecido nervoso resulta em aumento da sensibilidade aos estímulos dolorosos e também os estímulos inócuos passam a serem percebidos como dolorosos. Esta hiperalgesia neuropática pode ser devida, em parte, às alterações centrais ou espinhais, porém um componente relevante provavelmente seja a sensibilização dos aferentes primários periféricos (Tracey, Walker, 1995; Baron, 2000). Há evidências da participação do óxido nítrico (NO) na dor neuropática, uma vez que o NO poderia desempenhar um papel no processo de faci- 
litação espinhal pró-nociceptiva, contribuindo com a geração e manutenção da hiperalgesia neuropática (Yamamoto, Shimoyama, 1993; Meller et al., 1994). Perifericamente, no modelo de dor neuropática por constrição do nervo ciático (CNC), é observado aumento da síntese de NO nas fibras distais à lesão e no neuroma (Levy et al., 1999). Segundo George et al. (2005), há aumento dos níveis locais do fator de necrose tumoral (TNF- $\alpha$ ), a partir do primeiro dia pós-cirúrgico, o qual permanece até o vigésimo-oitavo dia. No segmento distal à lesão do nervo, ocorre também aumento significativo e de longa duração dos níveis de interleucina-1 $\beta$ (IL-1 $\beta$ ) e interleucina 10 (IL-10), provavelmente mediados pelos receptores NMDA (Kleinschnitz et al., 2004; Kleinschnitz et al., 2005).

A eficácia dos antiinflamátorios não-esteróides no tratamento da hipersensibilidade que se segue após a injúria de nervos periféricos é controversa, sendo as respostas clínicas consideradas modestas (Fields et al., 1999; MacPherson, 2002). No entanto, Marquez e Ferreira (1987) relataram que infusões locais de dipirona eram capazes de induzir analgesia de longa duração em pacientes portadores de dores neuropáticas intratáveis. Além disso, uma única administração intraplantar de dipirona também foi capaz de bloquear totalmente a hiperalgesia persistente induzida por $\mathrm{PGE}_{2}$ em ratos (Ferreira et al., 1990). Nos trabalhos citados anteriormente, a administração sistêmica de dipirona não foi efetiva para induzir efeito analgésico significativo nestes modelos de dor crônica.

Embora os fármacos antiinflamatórios e analgésicos não-esteróides atuem inibindo a ciclooxigenase e conseqüentemente a síntese de prostaglandinas (Vane, 1971) e deste modo possam aliviar significativamente as dores de origem inflamatória, este mecanismo de ação não parece efetivo no controle da dor neuropática (Fields et al., 1999). Entretanto, para a dipirona, tem sido sugerido mecanismo de ação analgésico distinto dos outros fármacos antiinflamatórios não-esteróides do tipo do ácido acetilsalicílico. A dipirona parece atuar exercendo bloqueio direto da hiperalgesia inflamatória por $\mathrm{PGE}_{2}$, supostamente promovendo dessensibilização dos nociceptores periféricos (Lorenzetti, Ferreira, 1985). Este mecanismo de dessensibilização, provavelmente envolve a ativação da via óxido-nítrico - GMPc no nociceptor (Duarte et al., 1992; Lorenzetti, Ferreira, 1996). A ação analgésica de diversos agentes, tais como a morfina, a nitroglicerina, o diclofenaco, a nimesulida, o meloxicam e o cetorolaco também parece depender da ativação endógena da via óxido-nítrico - GMPc para a dessensibilização do nociceptor (Ferreira et al., 1991; Ferreira et al., 1992;
Tonussi, Ferreira, 1994; Islas-Caderra et al., 1999; Aguirre-Bañuelos, Granados-Soto, 2000; Lázaro-Ibáñez et al., 2001).

Portanto, uma vez que o modelo de neuropatia por constrição do nervo ciático em ratos parece simular a condição clínica de compressão crônica de nervos, tais como as que ocorrem na neuropatia por aprisionamento do nervo ciático e seus ramos ou na irritação da raiz espinhal por hérnia de disco lombar (Zimmermann, 2001), o objetivo deste trabalho foi o de estabelecer o perfil analgésico da dipirona administrada localmente neste modelo de neuropatia e o possível envolvimento da ativação endógena e periférica da via NO-GMPc.

\section{MATERIAL E MÉTODOS}

Todos os procedimentos utilizados nos experimentos foram aprovados pela Comissão de Ética na Experimentação Animal (CEEA) do Instituto de Biociências, UNESP Botucatu, São Paulo, Brasil.

\section{Animais}

Ratos machos Wistar (UNESP, Brasil), pesando entre 180 e $200 \mathrm{~g}$. Os animais foram acondicionados em gaiolas $(n=4)$, mantidos em um ciclo claro escuro de 12 horas, temperatura ambiente de $25 \pm 1{ }^{\circ} \mathrm{C}$ e acesso à ração e água ad libitum.

\section{Procedimento cirúrgico}

A neuropatia foi induzida nos animais de acordo com o método descrito por Bennett e Xie (1988). Sob anestesia com pentobarbital sódico $(45 \mathrm{mg} / \mathrm{kg}$, intraperitoneal), o nervo ciático direito do rato foi exposto e um segmento de $5-7 \mathrm{~mm}$ foi dissecado. Quatro ligaduras com fio de sutura cat-gut cromado 4-0 tipo $\mathrm{C}$, frouxas o suficiente para não interromper a vascularização epineural superficial, com intervalos de $1 \mathrm{~mm}$ entre si, foram realizadas ao redor do nervo. $\mathrm{O}$ tecido incisionado foi suturado em camadas, com fio de sutura de algodão 40 . Em todos os animais, o nervo ciático do lado esquerdo também foi exposto e manipulado, porém não foram colocadas as ligaduras, sendo que este constituiu o lado controle da cirurgia (falso-operado).

\section{Teste nociceptivo}

O efeito antinociceptivo dos compostos testados foi avaliado através do teste de pressão na pata, descrito por Randall e Sellito (1957). Um aumento contínuo da pressão 
mecânica foi exercido pelo aparelho Basile Analgesymeter ${ }^{\circledR}$ (Ugo Basile, Itália), sobre o dorso da pata traseira do animal, até o reflexo de retirada ser observado. O limiar nociceptivo é definido como a força em gramas aplicada à pata do animal no momento em que este exibe o reflexo de retirada. O limiar sensorial basal foi mensurado em cada grupo de animais, imediatamente no pré-operatório e nos tempos de 7, 14, 21, 28 dias de pós-operatório. Os animais exibiram hiperalgesia mecânica já no sétimo dia de pósoperatório.

\section{Fármacos}

Os seguintes compostos foram testados: antiinflamatório não-esteróide: dipirona sódica (Dip) $\left(\right.$ Hoechst $\left.^{\mathbb{R}}\right)$; inibidor da óxido nítrico sintase (NOS), éster metílico da $\mathrm{L}^{\gamma}$ - nitro-L-arginina (L-NAME) (Sigma, St. Louis, MO). L-arginina (Sigma, St. Louis, MO); inativador do óxido nítrico endógeno, a hemoglobina $(\mathrm{Hb})$ (Sigma, St. Louis, MO); cloreto de metiltionínio (MB) (Merck) e a ${ }^{1} \mathrm{H}-[1,2,4]$ oxadiazol-[4,3-a]quinolaxin-1-one (ODQ) (Sigma, St. Louis, MO), dois inibidores da guanilil ciclase (GC); bloqueador de canais de potássio sensíveis ao ATP, glibenclamida (GLY) (Sigma, St. Louis, MO); doador de NO, o nitroprussiato de sódio (SNP) (Sigma, St. Louis, MO). Os fármacos foram dissolvidos em $\mathrm{NaCl}$ a $0,9 \%$, exceto a glibenclamida, a qual foi dissolvida em Tween 80 .

A administração dos fármacos foi realizada por via intra-plantar (i.pl.), na pata traseira direita dos animais. O volume administrado em todos os tratamentos foi de $0,1 \mathrm{~mL}$ pata $^{-1}$. Administrações de solução salina ou veículo foram realizadas nos grupos controles. Os tratamentos foram realizados no $14^{\circ}$ dia pós cirúrgico.

\section{Análise estatística}

Os resultados foram submetidos ao teste de Análise de Variância (ANOVA, para comparação de mais de duas amostras), seguidos do teste de Tukey-Kramer, para comparações múltiplas. Os dados são expressos como média \pm erro padrão da média. Os níveis de significância foram $* * p<0,01 \mathrm{e}^{*} \mathrm{p}<0,05$.

\section{RESULTADOS}

Após a cirurgia de constrição do nervo ciático, observa-se em todos os animais um aumento na intensidade de hiperalgesia mecânica, avaliada a partir do $7^{\circ}$ dia após a CNC. Este estado de hiperalgesia neuropática permanece por, no mínimo, 28 dias.
Efeito do bloqueio da síntese de NO sobre o desenvolvimento da analgesia de longa duração da dipirona em ratos com hiperalgesia neuropática

Uma única administração intraplantar de dipirona (80 $\left.\mathrm{mg} \mathrm{pata}^{-1}\right)$ (Ferreira et al., 1990) no $14^{\circ}$ dia após a CNC induziu um efeito analgésico significativo, de longa duração $(P<0,01)$ no $28^{\circ}$ dia após a CNC, em relação ao grupo con-

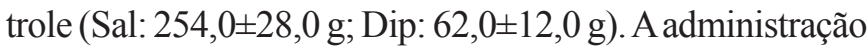
concomitante de L-NAME (50 - $100 \mu \mathrm{g} \mathrm{pata}^{-1}$ ) e dipirona (80 $\mathrm{mg} \mathrm{pata}^{-1}$ ), no $14^{\circ}$ dia após a $\mathrm{CNC}$, promoveu redução dose-dependente no desenvolvimento da analgesia de longa duração da dipirona, sendo observada redução significativa no $28^{\circ}$ dia após a CNC (Dip+L-NAME: $254,0 \pm 14,0$ g e Dip: $62,0 \pm 12,0 \mathrm{~g} ; P<0,01$ ). A administração de L-NAME (50 mg pata $\left.^{-1}\right)$ no $14^{\circ}$ dia após a CNC não alterou o desenvolvimento da hiperalgesia neuropática (Figura 1).

A L-arginina (500 $\left.\mu \mathrm{g}_{\text {pata }^{-1}}\right)$ bloqueou o efeito do L-NAME (50 mg pata-1 $)(P<0,05)$, possibilitando a instalação da analgesia de longa duração da dipirona (Dip: 114,0 $\pm 14,0$ g; Dip+L-NAME: 246,0 $\pm 18,0$ g; Dip+LNAME+L-Arg: $116,0 \pm 10,0$ g) (Figura 2). Os fármacos foram administrados concomitantemente no $14^{\circ}$ dia após a $\mathrm{CNC}$, pela via intraplantar. A administração de L-Arg (500 $\left.\mathrm{mg} \mathrm{pata}^{-1}\right)$ não alterou o desenvolvimento da hiperalgesia neuropática (Figura 2).

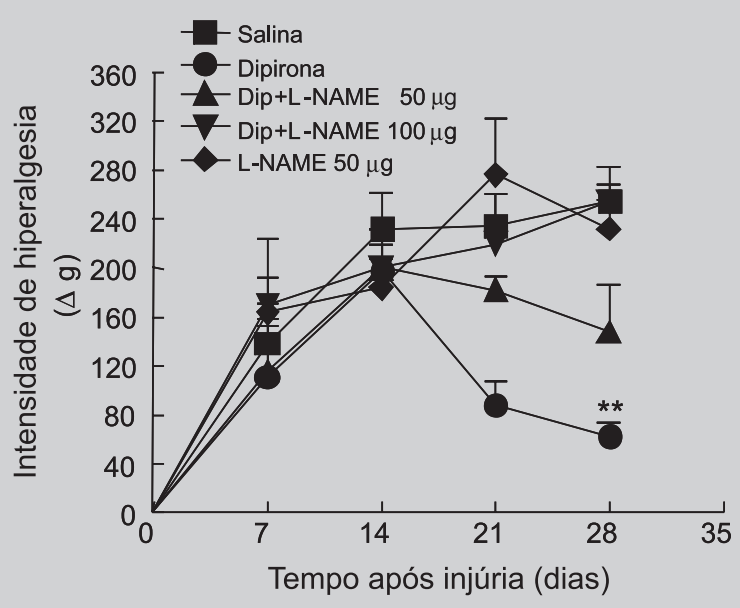

FIGURA 1 - Efeito da administração intraplantar de dipirona $\left(80 \mu \mathrm{g} \mathrm{pata}^{-1}\right) \mathrm{e} / \mathrm{ou}$ um bloqueador da NO sintase, L-NAME (50-100 $\mu \mathrm{g} \mathrm{pata}^{-1}$ ) sobre o desenvolvimento da hiperalgesia mecânica de ratos submetidos a $\mathrm{CNC}$ no teste de pressão da pata ( $\mathrm{n}=6$ por grupo). Os resultados são expressos como média \pm e.p. média. $* * P<0,01$; comparando-se o grupo tratado com dipirona com os grupos tratados com Dip $+\mathrm{L}-$ NAME $\left(100 \mu \mathrm{g} \mathrm{pata}^{-1}\right)$, com L-NAME $\left(50 \mu \mathrm{g} \mathrm{pata}^{-1}\right)$ somente e com salina $\left(0,1 \mathrm{~mL}\right.$ pata $\left.^{-1}\right)$. 


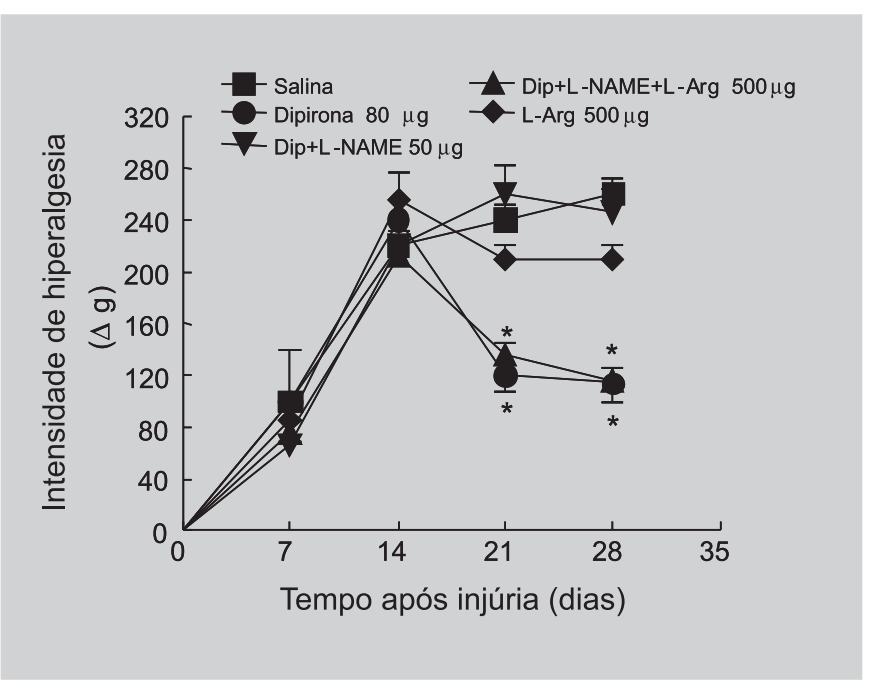

FIGURA 2 - Efeito da administração intraplantar concomitante de L-Arg $\left(500 \mu \mathrm{g} \mathrm{pata}^{-1}\right)$ e L-NAME $\left(50 \mu \mathrm{g} \mathrm{pata}^{-1}\right)$ sobre a antinocicepção induzida pela dipirona $\left(80 \mu \mathrm{g} \mathrm{pata}^{-1}\right)$ no desenvolvimento da hiperalgesia mecânica de ratos submetidos a CNC no teste de pressão da pata ( $\mathrm{n}=6$ por grupo). Os resultados são expressos como média \pm e.p. média. $* P<0,05$; comparando-se o grupo tratado com dipirona com os grupos tratados com Dip+L-NAME $\left(50 \mu \mathrm{g} \mathrm{pata}^{-1}\right)$ ou com salina $\left(0,1 \mathrm{~mL}\right.$ pata $\left.^{-1}\right)$ e os grupos tratados concomitantemente com L-Arg $\left(500 \mu \mathrm{g} \mathrm{pata}^{-1}\right) \mathrm{e}$ L-NAME $\left(50 \mu \mathrm{g} \mathrm{pata}^{-1}\right)$ com o grupo tratado com L-NAME $\left(50 \mu \mathrm{g} \mathrm{pata}^{-1}\right)$ ou com salina $\left(0,1 \mathrm{~mL} \mathrm{pata}^{-1}\right)$.

\section{Efeito da inativação do óxido nítrico endógeno extracelular periférico sobre o desenvolvimento do efeito analgésico de longa duração da dipirona em ratos com hiperalgesia neuropática}

A administração periférica (i.pl.) de hemoglobina (10 ou $30 \mathrm{mg} \mathrm{pata}^{-1}$ ) (Duarte et al., 1992) conjuntamente com a dipirona $\left(80 \mathrm{mg} \mathrm{pata}^{-1}\right)$, no $14^{\circ}$ dia após a $\mathrm{CNC}$, promoveu redução dose-dependente no desenvolvimento da analgesia de longa duração da dipirona (Sal: 248,0 $0 \pm 10,0 \mathrm{~g}$;

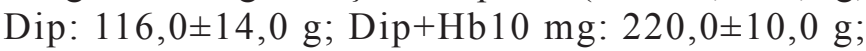
Dip+Hb30 mg: 230,0 $\pm 10,0 \mathrm{~g}$ ), sendo considerada significativa para a administração de $\mathrm{Hb}$ na dose de $30 \mathrm{mg}$ $(P<0,01)$ (Figura 3).

\section{Participação da ativação da via NO-GMPc no desenvolvimento da analgesia de longa duração da dipirona na hiperalgesia neuropática}

Ocorre significativa inibição no desenvolvimento da analgesia de longa duração da dipirona após o bloqueio da enzima guanilil ciclase e conseqüentemente da ativação da

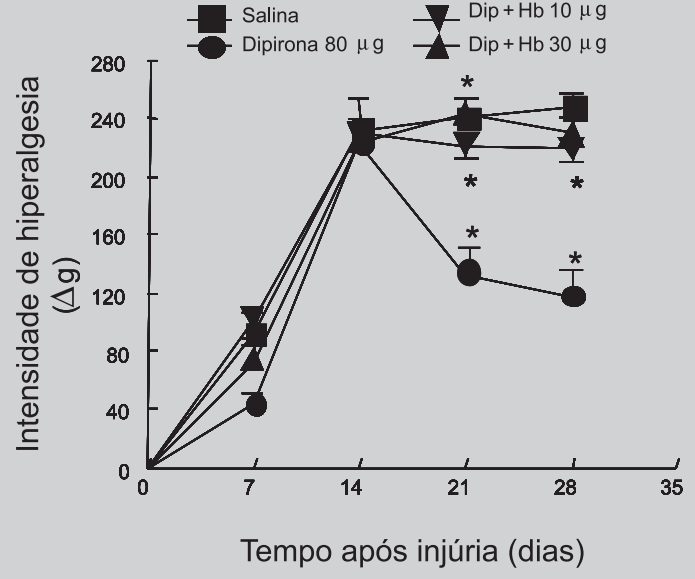

FIGURA 3 - Efeito da administração intraplantar de dipirona $\left(80 \mu \mathrm{g} \mathrm{pata}^{-1}\right)$ e ou um seqüestrante de $\mathrm{NO}, \mathrm{Nb}$ $\left(10\right.$ - $\left.30 \mu \mathrm{g} \mathrm{pata}^{-1}\right)$ sobre o desenvolvimento da hiperalgesia mecânica de ratos submetidos a CNC no teste de pressão da pata ( $\mathrm{n}=8$ por grupo). Os resultados são expressos como média \pm e.p. média. ${ }^{*} P<0,05$; comparando-se o grupo tratado com dipirona com os grupos tratados com $\mathrm{Dip}+\mathrm{Hb}$ $\left(10\right.$ - $30 \mu \mathrm{g}$ pata $\left.^{-1}\right)$.

via NO-GMPc. A administração concomitante de cloreto de metiltionínio (500 mg pata ${ }^{-1}$ ) (Lorenzetti, Ferreira, 1996) e dipirona ( $\left.80 \mathrm{mg} \mathrm{pata}^{-1}\right)$, no $14^{\circ}$ dia após a CNC (Figura 4), inibiu o desenvolvimento da analgesia de longa duração da dipirona, sendo observada redução significativa no $21^{\circ}$ dia após a CNC (Sal: 220,0 $\pm 30,0 \mathrm{~g}$ Dip+Cloreto de metiltionínio: $174,0 \pm 12,0$ g e Dip: 56,0 $\pm 14,0 \mathrm{~g} ; P<0,05)$. Além disso, a administração concomitante de ODQ (50 mg pata $\left.{ }^{-1}\right)$ (Lorenzetti, Ferreira, 1996) e dipirona (80 $\mathrm{mg} \mathrm{pata}^{-1}$ ), no $14^{\circ}$ dia após a CNC, também inibiu o desenvolvimento da analgesia de longa duração da dipirona (Figura 5), sendo observada redução significativa no $21^{\circ}$ dia após a CNC (Sal: 254,0 $\pm 18,0 \mathrm{~g}$ Dip+ODQ: $216,0 \pm 12,0$ g e Dip: $120,0 \pm 11,0 \mathrm{~g} ; P<0,05)$.

Efeito do bloqueio de canais de $\mathrm{K}^{+}$sensíveis ao ATP sobre o desenvolvimento da analgesia de longa duração da dipirona na hiperalgesia neuropática

Realizou-se nos animais com hiperalgesia neuropática pré-tratamento, no $14^{\circ}$ dia após a CNC, com glibenclamida $\left(100,200 \mathrm{ou} 300 \mathrm{mg} \mathrm{pata}^{-1}\right)$ (Rodrigues, Duarte, 2000), 5 minutos antes da administração de dipirona (80 $\mathrm{mg} \mathrm{pata}^{-1}$ ). A glibenclamida foi capaz de bloquear de forma dose dependente o desenvolvimento da 


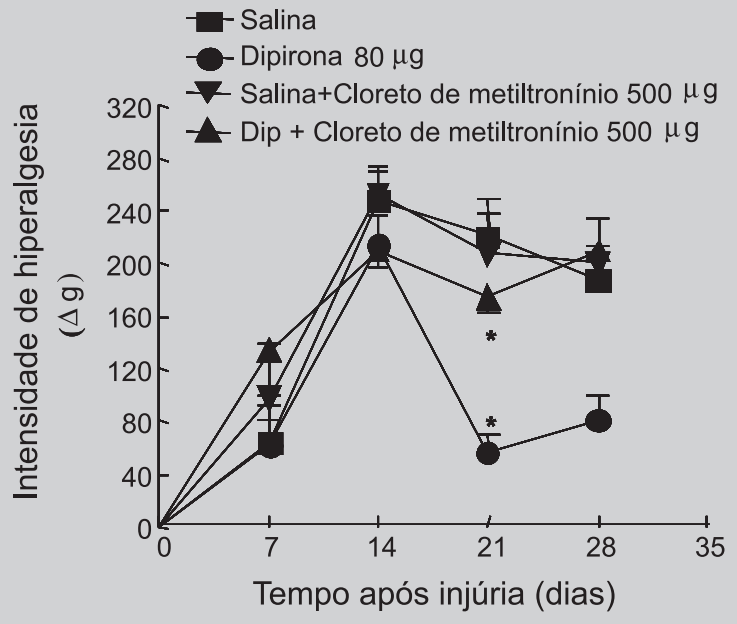

FIGURA 4 - Efeito da administração intraplantar de dipirona (80 $\mu$ g pata $\left.^{-1}\right)$ e/ou um inibidor da GC, azul de metileno (500 $\mu \mathrm{g}$ pata $^{-1}$ ) sobre o desenvolvimento da hiperalgesia mecânica de ratos submetidos a $\mathrm{CNC}$ no teste de pressão da pata $(\mathrm{n}=8$ por grupo). Os resultados são expressos como média \pm e.p. média. ${ }^{*} P<0,05$; comparando-se o grupo tratado com dipirona com os grupos tratados com Dip+cloreto de metiltronínio $\left(500 \mu \mathrm{g} \mathrm{pata}^{-1}\right)$, cloreto de metiltronínio $\left(500 \mu\right.$ g pata $\left.^{-1}\right)$ ou salina $\left(0,1 \mathrm{~mL}\right.$ pata $\left.^{-1}\right)$.

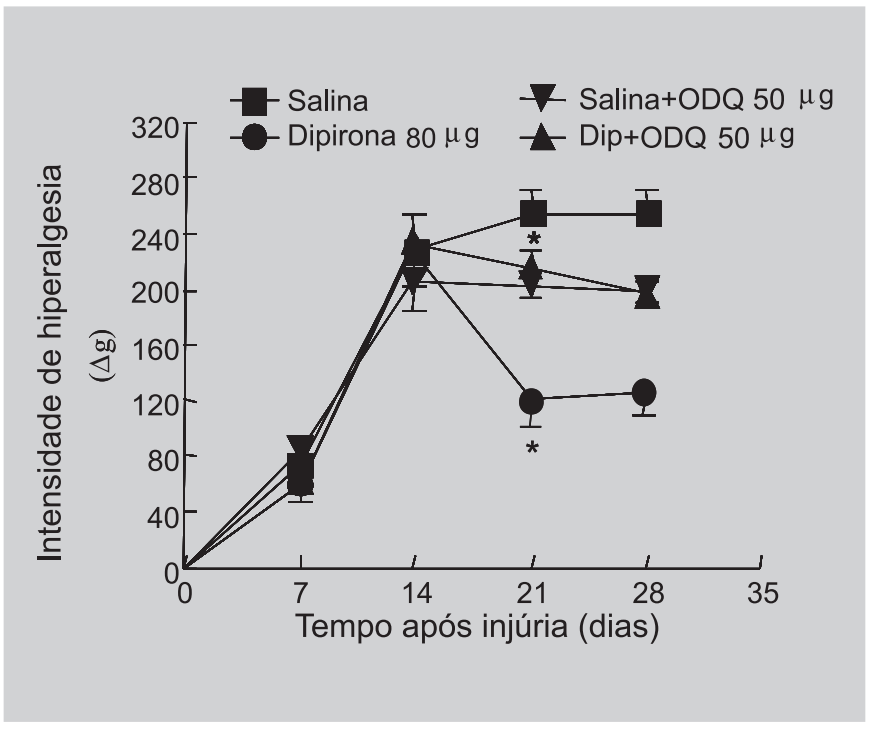

FIGURA 5 - Efeito da administração intraplantar de dipirona (80 $\left.\mu \mathrm{g} \mathrm{pata}^{-1}\right)$ e ou um inibidor da GC, ODQ $\left(50 \mu \mathrm{g} \mathrm{pata}^{-1}\right)$ sobre o desenvolvimento da hiperalgesia mecânica de ratos submetidos a $\mathrm{CNC}$ no teste de pressão da pata ( $\mathrm{n}=8$ por grupo). Os resultados são expressos como média \pm e.p. média. $* P<0,05$; comparando-se o grupo tratado com dipirona com os grupos tratados comDip+OQD $\left(50 \mu \mathrm{g} \mathrm{pata}^{-1}\right)$, OQD $\left(50 \mu \mathrm{g} \mathrm{pata}^{-1}\right)$ ou salina $\left(0,1 \mathrm{~mL}\right.$ pata $\left.^{-1}\right)$.

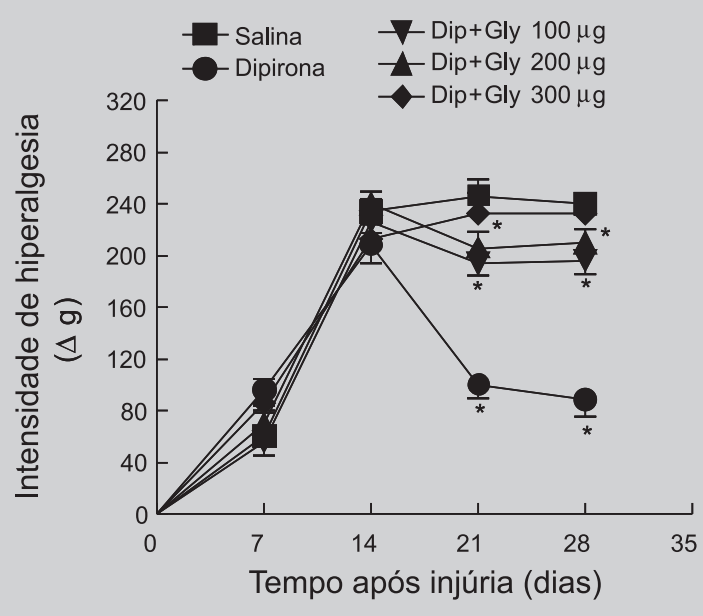

FIGURA 6 - Efeito da administração intraplantar de dipirona ( $\left.80 \mu \mathrm{g} \mathrm{pata}^{-1}\right)$ e/ou um bloqueador de canais de potássio sensíveis ao ATP, Gly (100-200-300 $\mu$ g pata $\left.^{-1}\right)$ sobre o desenvolvimento da hiperalgesia mecânica de ratos submetidos a CNC no teste de pressão da pata ( $\mathrm{n}=8$ por grupo). Os resultados são expressos como média \pm e.p. média. ${ }^{*} P<0,05$; comparando-se o grupo tratado com dipirona com os grupos tratados com Dip+Gly $(100,200$, $\left.300 \mu \mathrm{g} \mathrm{pata}^{-1}\right)$ ou com salina $\left(0,1 \mathrm{~mL}\right.$ pata $\left.{ }^{-1}\right)$.

analgesia de longa duração da dipirona (Sal: 240,0土8,0 g;

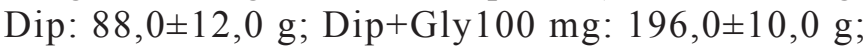
Dip+Gly200 mg: 210,0 $\pm 10,0$ g; Dip+Gly300 mg: $232,0 \pm 14,0 ; P<0,05$ ) (Figura 6).

\section{Efeito da administração local de um doador de óxido nítrico sobre a hiperalgesia mecânica neuropática}

Na Figura 7 observa que uma única administração local de SNP (500 $\mathrm{mg} \mathrm{pata}^{-1}$ ) (Soares et al., 2000), no $14^{\circ}$ dia após a CNC, induziu efeito analgésico significativo e de longa duração sobre a hiperalgesia mecânica neuropática, avaliado até o $28^{\circ}$ dia após a CNC (Sal: 220,0 $12,0 \mathrm{~g}$; SNP: $110,0 \pm 10,0 \mathrm{~g} ; P<0,05)$.

\section{DISCUSSÃO}

Os efeitos analgésicos, antiinflamatórios e antipiréticos dos fármacos antiinflamatórios não-esteróides são atribuídos à inibição da enzima ciclooxigenase e, portanto, ao bloqueio da síntese de prostaglandinas (Vane, 1971). Contudo, a possibilidade de um efeito central da dipirona em relação à inibição da síntese de $\mathrm{PGE}_{2}$ tem sido aventada (Shimada et al, 1994; Mccormack, Brune, 1991). 


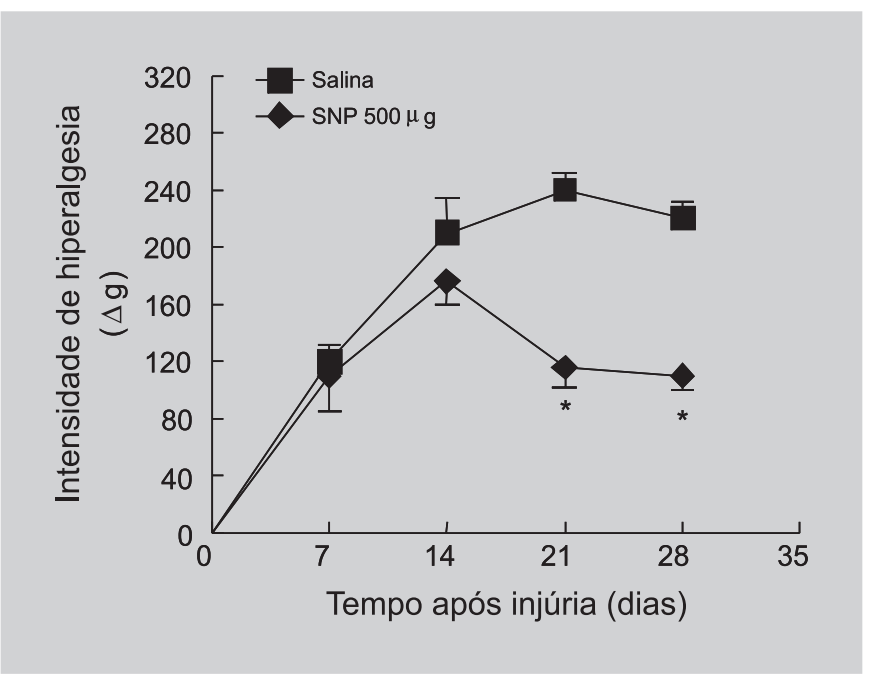

FIGURA 7 - Efeito da administração intraplantar de SNP $\left(500 \mu \mathrm{g} \mathrm{pata}^{-1}\right)$ sobre o desenvolvimento da hiperalgesia mecânica de ratos submetidos a CNC no teste de pressão da pata ( $\mathrm{n}=5$ por grupo). Os resultados são expressos como média \pm e.p. média. ${ }^{*} P<0,05$; comparando-se o grupo tratado com SNP com o grupo tratado com salina $(0,1 \mathrm{~mL}$ pata $\left.^{-1}\right)$.

No entanto, na neuropatia por secção parcial do nervo ciático, demonstrou-se a importância da prostaglandina $\mathrm{E}_{2}$ no desenvolvimento da hiperalgesia e alodinia. A administração intraplantar de indometacina, piroxicam ou antagonista de receptor $\mathrm{EP}_{1}$ até o sexto dia após a cirurgia (Syriatowicz et al.,1999) ou a administração intratecal de indometacina no primeiro dia após a cirurgia (Zhao et al., 2000) atenuaram a hiperalgesia mecânica/térmica e a alodinia, respectivamente. Entretanto, Zhao et al. (2000) não obtiveram efeito analgésico com a administração intratecal de indometacina no $14^{\circ}$ dia pós cirúrgico, ou seja, após o estabelecimento do quadro de alodinia. Estes autores sugerem um papel importante para os prostanóides apenas na fase de instalação (e não na fase de manutenção) da alodinia em que os níveis espinhais de ciclooxigenase2 estão elevados (até o terceiro dia). Mais recentemente, estes dados foram confirmados, uma vez que o piroxicam, administrado pela via intratecal, também foi ineficaz em reverter a alodinia já instalada, neste mesmo modelo experimental (Ma, Eisenach, 2002a).

Esta ação antinociceptiva, periférica e de longa duração de uma única dose de dipirona, observada neste trabalho, demonstra perfil analgésico diferente deste fármaco em relação aos demais fármacos antiinflamatórios/analgésicos não-esteróides.

Com relação ao possível mecanismo de ação analgésica da dipirona, Lorenzetti e Ferreira (1985) demons- traram o bloqueio direto da hiperalgesia inflamatória por $\mathrm{PGE}_{2}$, provavelmente decorrente da dessensibilização dos nociceptores periféricos previamente sensibilizados por este mediador. $\mathrm{Na}$ hiperalgesia inflamatória por carragenina, já foi demonstrado que a analgesia periférica induzida pela acetilcolina (Duarte et al., 1990), assim como pela dipirona (Duarte et al., 1992) na hiperalgesia por $\mathrm{PGE}_{2}$ em ratos, provavelmente seria mediada via liberação de NO.

Marquez e Ferreira (1987) descreveram efeito analgésico potente da dipirona sobre dor crônica, em um estudo clínico piloto. Dores crônicas intratáveis em humanos, predominantemente devido à desaferentação, foram aliviadas a partir de infusões regionais de dipirona, sendo que o efeito analgésico foi relevante e de longa duração, permanecendo por até dois meses.

Este possível mecanismo de dessensibilização dos nociceptores periféricos, independente da inibição da síntese de prostaglandinas, parece também ser responsável pela analgesia local induzida pelo diclofenaco (Tonussi, Ferreira, 1994) e pela morfina e $N$-metilmorfina na hiperalgesia aguda por $\mathrm{PGE}_{2}$ (Ferreira et al.,1990).

Similar ao verificado em nosso estudo, a dipirona e a $N$-metilmorfina administradas localmente, exercem efeito analgésico de longa duração sobre a hiperalgesia persistente por $\mathrm{PGE}_{2}$ (Ferreira et al., 1990). Estes mesmos autores também observaram que a morfina administrada sistemicamente no modelo experimental de hiperalgesia persistente por $\mathrm{PGE}_{2}$ apenas exerceu efeito transitório.

No modelo de alodinia induzida por secção parcial do nervo ciático, observou-se efeito analgésico de longa duração (5 dias) do cetorolaco intraplantar (Ma, Eisenach, 2002 a,b). No entanto, a administração sistêmica (ip ou im) deste antiinflamatório e analgésico induziu apenas analgesia de curta duração (Ma, Eisenach, 2002 b).

A inibição da síntese de NO com L-NAME, em doses que não alteraram o desenvolvimento da hiperalgesia neuropática por constrição do nervo ciático nos controles, reduziu de maneira dose-dependente a instalação do efeito analgésico de longo tempo da dipirona. Este efeito inibitório do L-NAME sobre a analgesia da dipirona foi bloqueado pela administração intraplantar concomitante de L-arginina. A L-arginina, por sua vez, não alterou a hiperalgesia neuropática persistente nos controles.

A hemoglobina $(\mathrm{Hb})$, um composto que atua inativando o NO endógeno extracelular gerado tanto pela NOSc ou NOSi, quando administrado concomitantemente à dipirona também bloqueou a instalação do efeito analgésico local e de longa duração da dipirona. 
Estes resultados corroboram a hipótese de que a síntese de NO, perifericamente, pode estar relacionada com o efeito analgésico local da dipirona, assim como já tem sido verificado para outros analgésicos em distintos modelos experimentais, tais como a própria dipirona e o mirceno na hiperalgesia aguda induzida por $\mathrm{PGE}_{2}$ (Duarte et al.,1992); o diclofenaco na hiperalgesia inflamatória (Tonussi, Ferreira, 1994); a morfina, na hiperalgesia por $\mathrm{PGE}_{2}$ e no teste da formalina (Ferreira et al., 1991;Granados-Soto et al., 1997); o meloxicam (Aguirre-Bañuelos, GranadosSoto, 2000) e o cetorolaco (Lázaro-Ibáñez et al., 2001), no teste da formalina.

Embora o NO, perifericamente, parece estar mediando a ação analgésica de diversos fármacos, a sua participação nos processos nociceptivos ainda não está clara. O bloqueio da síntese de NO na coluna espinhal e a conseqüente diminuição de sua concentração pode realçar o efeito central analgésico de agonistas opióides no teste da formalina em ratos (Machelska et al., 1997). Além disso, o L-NAME, um bloqueador da NOS, também pode evocar um efeito antinociceptivo direto no sistema nervoso central no teste da formalina em camundongos (Moore et al.,1991). Sugere-se, também, a participação do NO na hiperalgesia mecânica periférica induzida por bradicinina (Nakamura et al., 1996).

Yamamoto e Shimoyama (1993) demostraram que NO poderia desempenhar um papel no processo de facilitação espinhal observada no modelo de hiperalgesia neuropática induzida a partir da constrição do nervo ciático. Desta forma, a síntese de NO na medula espinhal e no sistema nervoso central estaria associada à nocicepção. Perifericamente, neste mesmo modelo de dor neuropática, é observado aumento da síntese de NO a partir da estimulação da enzima NOSi no neuroma e nas fibras distais à lesão, sugerindo-se participação do NO na patogênese da hiperalgesia neuropática (Levy et al., 1999).

Ainda com relação à participação do NO na dor neuropática, tem sido demonstrado que a sua produção espinhal contribui com a geração e manutenção da hiperalgesia térmica (Meller et al., 1996).

Entretanto, o papel do NO nos processos periféricos não está claro, uma vez que alguns autores relatam papel pró-nociceptivo (Meller, Gebhart, 1994), enquanto outros autores descrevem ação antinociceptiva. Neste sentido, Mashimo et al. (1995) relataram que a administração intravenosa de nitroglicerina pode aliviar a dor espontânea e a causalgia em humanos. No modelo de injúria por constrição do ciático em animais, a administração intratecal do $\mathrm{NO}$ em altas concentrações parece não exercer nenhum efeito ou pequeno efeito nociceptivo, enquanto em baixas concentrações induz diminuição da alodinia dinâmica (Souza, Prado, 2001).
No entanto, a L-arginina, o precursor da síntese de NO, parece possuir efeito dual no processo nociceptivo no SNC. Este composto poderia atuar como substrato na via quiotorfina - Met-encefalina, causando efeito antinociceptivo, ou atuar ativando a via $\mathrm{NO}-\mathrm{GMPc}$, realçando o processo nociceptivo ao nível central (Kawabata et al.,1993).

A liberação local de NO poderia ativar a enzima guanilil ciclase, aumentando a síntese de GMPc ao nível do nociceptor periférico (KAWABATA et al.,1993). Uma vez que a dipirona pode ativar a síntese de NO perifericamente, a ativação da via arginina - $\mathrm{NO}-\mathrm{GMPc}$ provavelmente seria responsável pelo efeito analgésico da dipirona (Duarte et al., 1992; Lorenzetti, Ferreira, 1996). De fato, a diminuição da síntese de GMPc com o bloqueio da enzima guanilil ciclase pelo cloreto de metiltionínio ou ODQ bloqueou o desenvolvimento do efeito analgésico de longa duração da dipirona.

Há evidências na literatura de que o NO pode ativar diferentes tipos de canais de potássio em diversos tecidos (Armstead, 1996; Edwards, Weston, 1993). Sugere-se que a ativação de canais de potássio sensíveis ao ATP, induzida pelo NO poderia ser mediada pelo aumento dos níveis de GMPc.

Alguns autores sugerem que a morfina, atuando em receptores opióides periféricos, também ative a via $\mathrm{NO}$ GMPc, induzindo efeito analgésico periférico (Duarte et al., 1990; Granados-Soto et al., 1997), o qual provavelmente seja devido a ativação de canais de potássio sensíveis ao ATP, uma vez que a glibenclamida, um bloqueador de canais de $\mathrm{K}^{+}$sensíveis ao ATP, inibe este efeito analgésico periférico da morfina (Rodrigues, Duarte, 2000). Em nosso trabalho, o desenvolvimento da analgesia de longa duração da dipirona também foi bloqueado pela glibenclamida.

Uma vez que a dipirona poderia atuar a partir da indução da geração espontânea local de NO, estudamos o efeito do nitroprussiato de sódio (NPS), um doador de NO, sobre a hiperalgesia neuropática por constrição do nervo ciático. O NPS, administrado pela via intraplantar, reduziu a hiperalgesia persistente por CNC. O efeito analgésico obtido com a administração local de NPS também foi de longa duração, sendo semelhante ao obtido com a dipirona.

Estes resultados confirmam outros dados da literatura referentes à ação analgésica periférica do nitroprussiato de sódio e de outros doadores de NO (Ferreira et al., 1992; Soares et al., 2000).

Nossos dados, portanto, nos permitem sugerir que o efeito analgésico periférico de longa duração da dipirona sobre a hiperalgesia persistente já instalada por constrição do nervo ciático, possa ser devido à ativação da via arginina - NO - GMPc - canais de $\mathrm{K}^{+}$sensíveis ao ATP, provavel- 
mente, induzindo dessensibilização dos terminais nociceptivos no neurônio sensorial primário. Este mecanismo pode ser também responsável pela manutenção deste quadro antinociceptivo de longa duração. Com relação à ação prolongada da dipirona, a duração desta analgesia periférica é muito maior do que o esperado, devido à meiavida curta ( 3 horas) deste fármaco no organismo. Este fato parece interessante do ponto de vista de uma possível utilidade clínica em dores crônicas.

\section{ABSTRACT \\ Long term analgesic effect of dipyrone on the persistent hyperalgesia induced by chronic constriction injury of sciatic nerve in rats: involviment of nitric oxide}

The long term analgesic effect of dipyrone was evaluated on a model of neuropathic pain and the role of nitric oxide/GMPc pathway in this antinociceptive mechanism. One intraplantar dipyrone administration (80 mg), at 14 th day after the chronic constriction injury of the sciatic nerve, induced a significant and long term analgesic effect. The inhibition of nitric oxide synthase (NOS) with L-NAME (50 or $100 \mathrm{mg} / \mathrm{paw}$ ) or scavenging of the endogenous $N O$ with hemoglobin $(10$ or $30 \mathrm{mg}$ / paw) inhibited the development of the dipyrone analgesia. L-arginine (500 $\mathrm{mg} / \mathrm{paw}$ ) could reverted the effect of L-NAME. Metylene blue (500 mg/paw) or ODQ (50 $\mathrm{mg} / \mathrm{paw})$ (blockers of guanyl cyclase), or glybenclamide (100, 200 or $300 \mathrm{mg} / \mathrm{paw}$ ) (blocker of ATP-sensitive $K^{+}$channels) inhibited the development of dipyrone analgesia. The sodium nitroprussiate administered at 14th day after the chronic constriction injury of the sciatic nerve also induced a long term analgesic effect similar to that of dipyrone. Our data may support the suggestion that the peripheral and the long term analgesic action of dipyrone on this model experimental occurs due to a probable nociceptor desensitisation with involviment of activation of the nitric oxide-cGMP pathway, followed by an opening of ATPsensitive $K^{+}$channels.

UNITERMS: Neuropathic hyperalgesia. Chronic constriction injury. Dipyrone. Nitric oxide.

\section{AGRADECIMENTOS}

Os autores agradecem à Coordenação de Aperfeiçoamento de Pessoal de Nível Superior-CAPES, pelo auxílio financeiro.

\section{REFERÊNCIAS BIBLIOGRÁFICAS}

AGUIRRE-BUÑUELOS, P.; GRANADOS-SOTO, V. Evidence for the paticipation of the nitric oxide-cyclic GMP pathway in the antinociceptive action of meloxicam in the formalin test. Eur. J. Pharmacol., v. 395, p. 9-13, 2000.

ARMSTED, W. M. Role of ATP sensitive $\mathrm{K}^{+}$channels in cGMP mediated pial artery vasodilation. Am. J. Physiol., v.270, p. H423-H426, 1996.

BARON, R. Peripheral neuropathic pain: from mechanisms to symptoms. Clin. J. Pain, v.16, n. 2, p.12-20, 2000.

BENNETT, G. J.; XIE, Y. K. A peripheral mononeuropathy in rat that produces disorders of pain sensation like those seen in man. Pain, v.33, p.87-107, 1988.

DEVOR, M.; SELTZER, Z. Pathophysiology of damaged nerves in relation to chronic pain. In: WALL, P. D.; MELZACK, R. (Eds). Textbook of pain. 4. ed. Edinburg: Churchill Livingstone, 1999. p. 59-84.

DUARTE, I. D.; LORENZETTI, B. B.; FERREIRA, S. H. Peripheral analgesia and activation of the nitric oxide cGMP pathway. Eur. J. Pharmacol., v.186, p.289-293, 1990.

DUARTE, I. D.; SANTOS, I. R.; LORENZETTI, B. B.; FERREIRA, S. H. Analgesia by direct antagonism of nociceptor sensitization involves the arginine - nitric oxide cGMP pathway.Eur.J.Pharmacol., v.217, p.225-227, 1992.

EDWARDS, G.; WESTON, A. H. The pharmacology of ATP - sensitive $\mathrm{K}^{+}$channels. Annu. Rev. Pharmacol. Toxicol., v.33, p.597-637, 1993.

FERREIRA, S. H.; LORENZETTI, B. B.; DE CAMPOS, D. I. Induction, blockade and restoration of a persistent hypersensitive state. Pain, v.42, p.365-371, 1990.

FERREIRA, S. H.; DUARTE, I. D.; LORENZETTI, B. B. The molecular mechanism of action of peripheral morphine analgesia: stimulation of the cGMP system via nitric oxide release. Eur. J. Pharmacol., v.201, p.207, 1991.

FERREIRA, S. H.; LORENZETTI, B. B.; FACCIOLI, L. H.. Blockade of hyperalgesia and neurogenic oedema by topical application of nitroglicerin. Eur. J. Pharmacol., v.217, p.207, 1992. 
FIELDS, H. L.; BARON, R.; ROWBOTHAM, M. C. Peripheral neuropathic pain: an approach to management. In: WALL, P. D.; MELZACK, R. (Eds). Textbook of pain. 4. ed. Edinburg: Churchill Livingstone, 1999. p.15231534.

GEORGE, A.; BUEHL, A.; SOMMER, C. Tumor necrosis factor receptor 1 and 2 proteins are differentially regulated during Wallerian degeneration of mouse sciatic nerve. Exp. Neurol., v.192 n. 1, p.163-166, 2005.

GRANADOS-SOTO, V.; RUFINO, M. O.; LOPES, L.D.G.; FERREIRA, S. H. Evidence for the involviment of the nitric oxide-cyclic GMP pathway in the antinociception of morphine in the formalin test. Eur. J. Pharmacol., v. 340, p. $177-180,1997$.

ISLAS-CADENA, M.; AGUIRRE-BANUELOS, P.; GRANADOS-SOTO, V. Evidence for the participation of nitric oxide-cyclic GMP pathway in the antinociceptive effect of nimesulide. J. Pharmacol. Toxicol., v.42, p.8792, 1999.

KAWABATA, A.; UMEDA, N.; TAKAGI, H. L-Arginine exerts a dual role in nociceptive processing in the brain: involvement of the Kyotorphin-Met-enkephalin pathway and NO-cyclic GMP pathway. Br. J. Pharmacol., v. 109, p. 73-79, 1993.

KLEINSCHNITZ, C.; BRINKHOFF, J.; ZELENKA, M.; SOMMER, C.; STOLL, G. The extent of cytokine induction in peripheral nerve lesions depends on the mode of injury and NMDA receptor signalling. $J$. Neuroimmunol., v. 149, n.12, p.77-83, 2004.

KLEINSCHNITZ, C.; BRINKHOFF, J.; SOMMER, C.; STOLL, G. Contralateral cytokine gene induction after peripheral nerve lesions: dependence on the mode of injury and NMDA receptor signalling. Brain Res. Mol., v. 136, n. 12, p. 23-28, 2005.

LÁZARO-IBÁÑEZ, G. G.; TORRES-LÓPES, J. E.; GRANADOS-SOTO, V. Participation of the nitric oxide - cyclic GMP-ATP-sensitive $\mathrm{K}^{+}$channel patway in the antinociceptive action of ketorolac. Eur. J. Pharmacol., v. 426, p. 39-44, 2001.

LEVY, D.; HÖKE, A.; ZOCHODNE, D. W. Local expression of the inducible nitric oxide synthase in an animal model of neuropathic pain. Neurosci. Lett., v. 260, p.207-209, 1999.
LORENZETTI, B. B.; FERREIRA, S. H. Mode of analgesic action of dipyrone : direct antagonism of inflammatory hyperalgesia. Eur. J. Pharmacol., v.114, p.375-381, 1985.

LORENZETTI, B. B.; FERREIRA, S. H. Activation of the arginine - nitric oxide pathway in primary sensory neurones contributes to dipyrone - induced spinal and peripheral analgesia. Inflamm. Res., v.45, p.308-311, 1996.

MA, W.; EISENACH, J. C. Role for both spinal cord COX1 and COX-2 in maintenance of mechanical hypersensitivity following peripheral nerve injury. Brain Res., v.937, p. 94-99, 2002 a.

MA, W.; EISENACH, J. C. Morphological and pharmacological evidence for the role of peripheral prostaglandins in the pathogenesis of neuropathic pain. Eur. J. Neurosci., v.15, p. 1037-1047, 2002 b.

MACHELSKA, H.; LABUZ, D.; PRZEWLOCKI, R.; PRZEWLOCKA, B. Inhibition of nitric oxide syntase enhances antinociception mediated by Um, delta and Kappa opioid receptors in acute and prolonged pain in the rat spinal cord. J. Pharmacol. Exp. Ther., v. 282, p. $977-$ 984, 1997.

MacPHERSON, R. D. New directions in pain management. Drugs Today, v.38, p. 135-145, 2002.

MARQUEZ, J. O.; FERREIRA, S. H. Regional dipyrone nociceptor blockade: a pilot study. Braz. J. Med. Biol. Res., v.20, p.441-444, 1987.

MASHIMO, T.; PAK, M.; INAGAKI, Y.; YOSHIYA, I. Effects of intravenous regional administration of vasodilators: guanethidine, nicardipine, nitroglicerine and prostaglandin E2 in a patient with causalgia. Pain Clin., v.8, p.255-261, 1995

McCORMACK, K.; BRUNE, K. Dissociation between the antinociceptive and anti - inflammatory effects of the nonsteroidal anti - inflammatory drugs. Drugs, v. 41, p. 533-547, 1991.

MELLER, S. T.; CUMMINGS, C. P.; TRAUB, R. J.; GEBHART, G. F. The role of nitric oxide in the development and maintenance of the hyperalgesia produced by intraplantar injection of carrageenan in the rat. Neuroscience, v. 60, p. 367-374, 1994. 
MELLER, S. T.; GEBHART, G. F. Nitric oxide (NO) and nociceptive processing in the spinal cord. Pain, v. 52, p. 127-136, 1993.

MOORE, P. K.; OLUYOMI, A. O.; BABBEDGE, P.; WALLACE, P.; HART, S. L. L-N-nitro arginine methyl ester exehibits antinociceptive activity in the mouse. Br. J. Pharmacol., v. 102, p. 198-202, 1991.

NAKAMURA, A.; FUJITA, M.; SHIOMI, H. Involvement of endogenous nitric oxide in the mechanism of bradikinininduced peripheral hyperalgesia. Br. J. Pharmacol., v. 117, p. 407-412, 1996.

RANDALL, L.; SELITTO, J.J. A method for the measurement of analgesic activit on inflamed tissue. Archs. Int. Pharmacodyn., v.111, p.409-419, 1957.

RODRIGUES, A. R.; DUARTE, I. D. G. The peripheral antinociceptive effect induced by morphine is associated with ATP-sensitive $\mathrm{K}^{+}$channels. Br. J. Pharmacol., v. 127, p.110-114, 2000.

SHIMADA, S. G.; OTTERNESS, I. G.; STITT, J. T. A study of the mechanism of action of the mild analgesic dipyrone. Agents Actions, v.41, p.188-192, 1994.

SOARES, A. C.; LEITE, R.; TATSUO M. A. K. F.; DUARTE, I. D. G. Activation of ATP-sensitive $\mathrm{K}^{+}$ channels: mechanism of peripheral antinociceptive action of the nitric oxide donor, sodium nitroprusside. Eur. $J$. Pharmacol., v.400, p.67-71, 2000.

SOUZA, A. M.; PRADO, W. A. The dual effect of a nitric oxide donor in nociception. Brain Res., v. 897, p. 9-19, 2001.
SYRIATOWICZ, J.; HU, D.; WALKER, J. S.; TRACEY, D. J. Hyperalgeis due to nerve injury: role of prostaglandins. Neuroscience, v. 94, p. 587-594, 1999.

TONUSSI, C. R.; FERREIRA, S. H. Mechanism of diclofenac analgesia: direct blockade of inflammatory sensitization. Eur. J. Pharmacol., v. 251, p. 173-179, 1994.

TRACEY, D. J.; WALKER, J. S. Pain due to nerve damage: are inflammatory mediators involved? Inflamm. Res., v. 44, p. 407-411, 1995.

VANE, J. R. Inhibition of prostaglandin synthesis as a mechanism of action for aspirin-like drugs. Nat., New Biol., v.231, p.232-235, 1971.

YAMAMOTO, T.; SHIMOYAMA, N.; MIZUGUCHI, T. Role of the injury discharge in development of thermal hypersthesia after sciatic nerve constriction injury in the rat. Anesthesiology, v.79, p.993-1002, 1993.

ZHAO, Z.; CHEN, S. R.; EISENACH, J. C.; BUSIJA, D. W.; PAN, H. L. Spinal cyclooxygenase- 2 is involved in development of allodynia after nerve injury in rats. Neuroscience, v.97, p.743-748, 2000.

ZIMMERMANN, M. Pathobiology of neuropathic pain. Eur. J. Pharmacol., v.429, p.23-37, 2001.

Recebido para publicação em 28 de setembro de 2005 Aceito para publicação em 19 de novembro de 2006 